\title{
V. \\ Ueber das Age oder Axin, ein in Mexico bereitetes trocknendes Fett.
}

\author{
Von \\ Dr. Felix Hoppe.
}

Vom königl. preuss. Marinearzte, Herrn Dr Friedel, erhielt ich im Herbste des vorigen Jahres eine etwa $50 \mathrm{Grm}$. betragende Quantität eines gelben aussen getrockneten Fettes in Palmenblätter verpackt, mit der Aufforderung, diese Substanz, welche in Mexico unter dem Namen Age oder Axin medicinisch angewendet werde, chemisch zu untersuchen. Die Litcratur der Chemie und Pharmakologie durchsuchte ich vergebens nach Andeutungen über dieses eigenthümliche Fett, und da nicht allein die chemische Constitution desselben manches Interessante bietet, sondern auch die technische und medicinische Verwendung desselben grosse Vortheile verspricht, so hat Herr Friedel mir dasjenige, was er über Ursprung, Gewinnung, medicinische Anwendung in Erfahrung gebracht hat, zur Veröffentlichung übergeben, und ich habe daran die Mittheilung der von mir bis jetzt gefundenen chemischen Verhältnisse dieser ausserordentlich zersetzlichen Sulsstanz geknüpft. Herr Friedel schreibt mir Folgendes :

Als ich im Anfange März 1858 mit S. M. Fregatte Gefion nach Mexico kam, hatte ich das Vergnügen, in in Vera Cruz die Bekanntschaft des daselbst ansässigen Dr. Behrendt zu machen. Unter vielen schätzenswerthen Mittheilungen, welche er mir machte, erhielt ich auch den hier in Rede stehenden Körper zum Geschenk. Seinem Wunsche gemäss theile ich Ihnen denselben mit, da mir die Mittel zu einer genaueren chemischen Untersuchung fehlen.

Die mexicanische Pharmacopoë vom Jahre 1846 führt nämlich sub litt. A folgendes auf: „Age oder Axin. Eigen- 
thümliche salbenartige Substanz, gewonnen aus dem Coccus axin La Slave's. Tonicum Catagmaticum."

Dieses Age ist nun eine dunkelgelbe butterähnliche Substanz, die in kleinen länglich-viereckigen Packeten, 2 bis 3 Mal in getrocknete, ausgebleichte Pisang-Blätter eingewickelt und überkreuzt mit den rothen Bastfaden der Yucca aloifolia zugeschnürt, in die mexicanischen $\Lambda$ potheken geliefert werden. Der Ursprung dieser Substanz ist eben so eigenthümlich wie ihre sonstigen Eigenschaften. Auf mehreren harzigen und balsamischen Bäumen lebt nämlich in den heissen und gemässigten Strichen Mexicos eine Coccusart, die nach Art der Cochenille-Schildlaus cultivirbar ist. Sie erreicht ausgewachsen die Grösse eines Haselnusskernes und zeigt eine matte, röthlich-graue Farbe. La Slave (spanischer Missionär?), hat diesen Coccus nach seinem einheimischen Namen mit der Speciesbezeichnung axin genannt.

Die Exemplare, die mir davon zu Gesicht gekornmen sind, waren theils in Spiritus aufbewahrt, theils getrocknet. Später beobachtete ich Legionen von eben ausgeschlüpften jungen Thieren. Ich hatte nämlich eine Schachtel voll der in dichte, feine, von den Mutterthieren abgestreifte, Wolle eingebetteten Eier mitgenommen, um sie zum Auskriechen etc. $\mathrm{zu}$ bringen. Sie waren von der Grösse eines kleinen, länglichen Stecknadelknopfes, oval und theils hellgelb, theils rosaroth gefärbt. Ob diese Verschiedenheit der Farbe durch Geschlechtsverschiedenheit bedingt wurde, vermag ich nicht anzugeben. Auf der Rückreise öffnete ich die Schachtel verschiedene Male, ohne eine Veränderung an dem Inhalt wahrzunehmen. Im Edinburgher Hafen bedeckten sich plötzlich die Wände meiner Kammer über und über mit blattlausartigen Thieren, Flüchtlingen aus jener Schachtel, und ehe ich Gelegenheit hatte, sie einzufangen und zu beobachten, hatten diensteifrige Hände alle Thierchen hinausgefegt.

Die Indianer Mexicos sammeln nun die in förmlichen Plantagen gezogenen grossen Thiere ein, tödten sie in heissem Wasser und schöpfen das aus der, Thieren herausgetretene Fett ab, kneten es erkaltet in $2^{\prime \prime}$ lange, $1 \frac{1}{2}{ }^{\prime \prime}$ breite 
und 1" hohe Stückchen. Anfünglich bleibt die Masse aussen weich und butterartig; durch Liegen an der Luft verwandelt sich aber später ihre Rinde in eine orangerothe harte Kruste, welche circa 1 Linie dick wird und dann die weitere Lufteinwirkung abschneidet.

Das Fett hat einen angenchmen, an die Arnicablüthen erinnernden. Geruch und verbindet in chirurgischer IIinsicht die Yorzüge des Collodium, des Traumaticin und den einer kühlenden Salbe in sich. Das eigenthümliche schnelle Erhärten der Substanz an der Luft und die Verwandlung einer auf die Haut aufgetragenen Schicht derselben in eine nach $A$ rt des Collorliums haftende und sich contrahirende, elastische Membran, die man zu beliebiger Dicke erhöhen kann, ohne dass sie dabei aufblättert, und die elastisch bleibt, sichert ihr diese Stellung z.lu.

Die Mexicaner wenden desshalb auch das $\Lambda$ ge in allen den Fällen an, wo es ihnen darauf ankommt, von Eruptionen aller $\Lambda \mathrm{rt}$ hefallene Hautpartien, Brandwunden, Geschwürc, Extravasate, contundirte . Stellen, Drüsengeschwïlste, rheumatische $\Lambda$ nschwellungen der Gelenke, locale Muskelrheumen mit einer milden, allmählich comprimirenden, luftabschliessenden Decke zu umgeben. Sie benutzen es ferner als Retentions- und Ifeilmittel bei entstehenden Hernien, indem sie die Haut über der Bruchpforte so lange damit bestreichen, bis sie sich sternförmig contrahirt und den Bruch zurïckhält. Auch bei leichten Fracturen, ohne Neigung zur Iislocation der Bruchenden, soll es gute Dienstc leisten. Vielleicht könnten kurze Bindenstücke damit bestrichen, einen eben so guten und weniger umständlichen Verband für die Fracturen greben, wie die Gypsbinden. Bei Varicositäten an den Unterschenkeln liessen sich ebenfalls gute Resultate erwarten.

Technisch ist es ein vorzüglicher Firniss für feine Stahlinstrumente gegen Rost.

Die Bäume, auf denen das Thier lebt, nennen die Mexicaner Palo mulato und Ciruela. Jenes ist eine Species ron Schimus; ob sie identisch ist mit Sch. molle $L$. oder dessen Var. $\beta$. Arrira kann ich nicht mit Bestimmtheit behaupten. Den botanischen Namen der 2. Gattung kann 
ich nicht ausfindig machen, da ich denselben nicht gesehen habe.

Merkwürdig bleibt es vom chemischen Standpunkte, dass sich dieses eigenthümliche Product von einem Thier herschreibt, welches auf einer Pflanze einer Familie lebt, die durch viele ihrer Mitglieder besondere Producte erzeugt. Zu den Cassavicae $\boldsymbol{R}$. Br. gehören bekanntlich Anacarrimum orient. und occid. (Acajou-Nüsse liefernd), Pistacia terebinthima (cypr. Terpentin), Pistacia mastix (Mastix), Rhus vernicifera (japan. Firniss), Rh. succedanea (japan. Wachs), Rh. copallinum (Copal und Anime). Die nahestehende Familie der Amyrielleae liefert das Flemi, Tacamahaca, Myrrhe, Meccabalsam, Olibanum, alles Substanzen, deren Vergleichung mit dem Age Stoff $z, u$ interessanten Untersuchungen geben würde. Eben so interessant würde es sein, eine vergleichende Untersuchung der Producte der Thiere aus der Familie Homoptra anzustellen, wie der Cochenille, des Gummilack, des Johannisblut (von Cocc. polonicus), der Absonderungen, welche die Arten von Lecaninm, Chermes, Lachinis, Aphis elc. von sich geben."

Das von Herrn Friedel mir übergebene Age zeigte einen leichten etwas an Firnisse erinnernden Geruch, war völlig unlöslich in Wasser, schwer löslich in kaltem, viel leichter in heissem Alkohol, sehr löslich in Aether, bis auf die erhärtete Kruste der Oberläche des Stückes, welche in allen jenen Flüssigkeiten unverändert blieb. Da Stücke des Fettes an der Oberläche schnell erhärteten, wenn sie sich in Berührung mit atmosphärischer Luft befanden, so wurde die ätherische Lösung des Fettes aus einem Kolben durch Destillation von $\Lambda$ ether befreit und im trocknen Wasserstoffstrome völlig getrocknet. Der Rückstand war eine bei $31^{\circ}$ schmelzende und erstarrende Substanz won brauner Farbe, deren Schmelzpunkt sich niedriger zeigte, wenn sie längere Zeit auf dem Wasserbade bei einer Temperatur von nahezu $100^{\circ}$ erhalten war.

0,4087 Grm. des Fettes der Einwirkung der atmosphärischen Luft einigre Tage über ausgesetzt (unter häufigem Auftröpfeln von etwas $A$ ether zur Erneuerung des Zutrittes der atmosphärischen Luft $\mathrm{zu}$ dem noch unzer- 
setzten Fette lieferten 0,4550 Grm. Gemenge von Stoffen, welche nur theilweise in Aether löslich waren; $0,295 \mathrm{Grm}$. in Aether unlöslicher Stoffe war darin enthalten. Durch Einwirkung der atmosphärischen Luft auf das Fett hatte dieses somit um 11,33 p.C.an Gewicht zugenommen. Dieselbe Veränderung des Fettes bewirkte Sauerstoff aus chlorsaurem Kali dargestellt unter Abnahme des Sauerstoffvolumen. Die Analyse *) des im Wasserstoffstrome getrockneten vor atmosphärischer Luft möglichst bewahrten Fettes gab folgende Werthe:

I. 0,2325 Grm. Fett gab 0,2180 Grm. Wasser und $0,6675 \mathrm{Grm}$. Kohlensäure.

II. 0,2460 Grm. Fett gab 0,2225 Grm. Wasser und 0,6980 Grm. Kohlensäure.

III. 0,1810 Grm. Fett gab 0,1720 Grm. Wasser und $0,518 \check{3}$ Grm. Kohlensäure.

IV. 0,1855 Grm. Fett gab $0,1733 \mathrm{Grm}$. Wasser und 0,5325 Grm. Kohlensäure.

Hieraus ergiebt sich die Zusammensetzung:

$\begin{array}{cccr}\text { I. } & \text { II. } & \text { III. } & \text { IV. } \\ \text { p.C. } & \text { p.C. } & \text { p... } & \text { p.C. } \\ \mathrm{C}=\mathbf{7 8 , 3 2} & \mathbf{7 7 , 3 8} & \mathbf{7 8 , 1 2} & \mathbf{7 8 , 2 9} \\ \mathrm{H}=\mathbf{1 0 , 4 2} & \mathbf{1 0 , 0 5} & \mathbf{1 0 , 5 6} & \mathbf{1 0 , 3 3}\end{array}$

Bei der trocknen Destillation des Fettes zeigten sich Acroleïndämpfe; die Verseifung des Fettes mit alkoholischer Kalilauge ging schnell vor sich, beim Erkalten der alkalischen Lösung bildete sich ein gallertartiger, nach dem Abfiltriren und Trocknen pulvriger Niederschlag von hellgelber Farbe, der sich leicht in Wasser oder kochendem Alkohol, ziemlich schwer in kaltem Alkohol löste. Er wurde durch Lösen in heissem Alkohol und Erkaltenlassen gereinigt, dann in kochender alkoholischer Lösung mit einer heissen alkoholischen Lösung von essigsaurem Baryt zersetzt. Es entstand ein hellgelber, feinkörniger Niederschlag, bestehend aus einem Barytsalze, welches

* Die sämmtlichen folgenden Analysen sind im Luft- und Sauerstoffstrome unter Vorlegung von Kupferoxyd im Platinschiffeben ausgefübrt. 
nicht ohne Zersetzung schmelzbar war, sich bei $100^{\circ}$ gut trocknen liess und bei der Analyse folgende Werthe gab:

I. 0,381 Grm. Substanz gab 0,1615 Grm. schwefelsauren Baryt.

II. 0,303 Grm. Substanz gab 0,2395 Grm. Wasser, $0,6120 \mathrm{Grm}$. Kohlensäure und 0,1285 $\mathrm{Grm}$. schwefelsauren Baryt.

III. 0,310 Grm. Substanz gab 0,2370 Grm. Wasser, $0,6163 \mathrm{Grm}$. Kohlensäure und 0,1290 $\mathrm{Grm}$. schwefelsauren Baryt.

IV. 0,305 Grm. Substanz gab 0,2350 Grm. Wasser, $0,6050 \mathrm{Grm}$. Kohlensäure und 0,128 $\mathrm{Grm}$. schwefelsauren Baryt.

Hieraus ergeben sich:

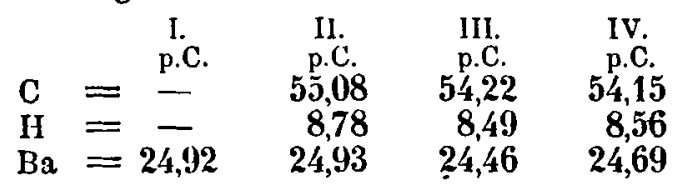

Das Kalisalz, aus dessen einer Portion das eben beschriebene Barytsalz erhalten war, mit Salzsäure zersetzt, gah eine schwach gelb gefärbte, an der Luft unveränderliche, in Wasser unlösliche, in Alkohol oder $\Lambda$ ether leicht lösliche, aus der heiss gesättigten alkoholischen Lösung beim Erkalten in mikroskopischen Nadeln sich ausscheidende feste Fettsäure, welche bei $42^{\circ}$ schmolz und erstarrte. Diese Eigenschaften so wie die Zusammensetzung des Barytsalzes lassen kaum einen $\mathbf{Z}$ weifel, dass diese Säure mit der Laurylsäure, $\mathrm{C}_{24} \mathrm{HI}_{24} \mathrm{O}_{4}$, identisch ist.

Der laurylsaure Baryt erfordert nach seiner Formel die procentische Zusammensetzung:

$$
\begin{aligned}
& \mathrm{C}=53,81 \text { p.C. } \\
& \mathrm{H}=8,59 "
\end{aligned}
$$

Die Vergleichung der berechneten mit den oben angeführten beobachteten Werthen ergiebt, dass in dem untersuchten Barytsalze etwas zu wenig Baryum und dem entsprechend zu viel Kohlenstoff gefunden ist, eine noch 
vorhandene Verunreinigung des Salzes durch eine Säure von höherem $\Lambda$ tomgewichte somit wahrscheinlich ist; hierfür spricht zufolge der Untersuchungen von $\mathrm{H}$ eintz auch der etwas zu niedrig gefundene Schmelzpunkt der Süure. Zur vollständigen Reinigung durch die fractionirte Fällung fehlte es mir an der nöthigen Quantität der Substanz, doch gelang es durch Behandlung mit nicht viel kochendem $\mathrm{Al}$ kohol aus einer Portion der kalisalze einen besonders schwer löslichen Theil desselben in geringer Quantität zu isoliren, welcher nach Zersetzung durch Salzsïure eine Säure lieferte, deren Schmelzpunkt ungefähr bei $60^{\circ}$ war, so dass neben der Laurylsäure etwas Palmitinsäure oder Stearinsäure im Age anzunehmen ist.

Die alkalische alkoholische Lösung, aus welcher sich beim Erkalten laurylsaures Kali niedergeschlagen hatte. lieferte nach Zusatz von Salzsäure einen dicköligen Niederschlag. welcher durch Zusat\% von Wasser noch sebr vermehrt wurde. Durch Decantiren und Waschen des Nicderschlags mit kaltem verdünnten $\Lambda$ lkohol wurde der Niederschlag getrennt und gereinigt, dann in $\Lambda$ ether gelöst, filtrirt, der Aether abdestillirt und der ölige Rückstand im Wasserstoffstrome auf dem Wasserbade getrocknet. El crstarrte noch über $\mathbf{0}^{0}$, zeigte braune Farbe und Geruch ähnlich dem Leinölfirniss, und in 3 Analysen eine Zusammensetzung, welche fast genau der Leinölsäure nach Schüler's*) L'ntersuchungen entsprach. Da aber offenbar bei der Darstellung der Sauerstoff der Luft auf diese Substanz sehr cnergisch eingewirkt hatte, so sehr sie auch vor Berührung der Luft in Acht genommen war, so wiederholte ich diese Darstellung mit einer anderen Portion Age nur mit der Abänderung, dass die sämmtlichen Proceduren, Lösen im Aether, Filtriren. Auswaschen, Verseifung $u$. s. w. im Wasserstoffstrome vorgenommen wurden, so dass höchstens durch unisemerkbare Undichtheit der Korke und Kautschukröhren oder beim Anfügen der-

-) Ann. der Chem. u. Pharm. Cl, 252. 
selben an Glasröhren (die jedoch so wie alle anderen Apparate vorher mit Wasserstoff gefüllt waren) Spuren von atmosphärischer Luft während der Behandlung das Age und die hervorgerufenen Producte treffen konnte. Die Filtration wurde in einem ganz geschlossenen Apparate vorgenommen und durch den Druck des Wasserstoffgases die Lösungen auf das Filter so wie auch bei den übrigen Proceduren aus einem Gefäss in das andere gebracht. Die schliesslich hierbei erhaltene Säure wurde nach dem Trocknen bei $100^{\circ}$ durch den Wasserstoffstrom nach Umdrehung der Richtung desselben, die er während des Trocknens gehabt hatte, in ein enges Glasrohr aufgetrieben, welches bis auf den Boden des Kolbens reichte, darauf wurde zuerst oben diess Rohr fest verschlossen, der Kolben mit dem Rohre schnell horizontal gelest, Kork und Rohr abgenommen und auch unten das Rohr mit einem Korke geschlossen. Die so erhaltene Säure ist dickflüssig ölig, bei $0^{0}$ noch nicht erstarrt, von brauner Farbe, unlöslich in Wasser, ziemlich löslich in kaltem, sehr leicht in heissem Alkohol oder Aether. In Berührung mit Sauerstoff gebracht überzieht sich die Oberfäche jedes Tropfens binnen 1 bis 2 Minuten mit einem feinen Häutchen, welches die Substanz im Innern vor der weiteren Einwirkung des Sauerstoffs schützt; bringt man wasserhaltigen Aether auf das erhärtete Häutchen, so wird es runzelig durch Aufnahme von Wasser und Aether. $Z u$ den folgenden Analysen wurden die ersten 3 aus der erwähnten Röhre entnommenen Proben benutzt; sofort nach Einbringung in das Platinschiffchen und schneller Wägung wurde die Probe in die Verbrennungsröhre gebracht und die Verbrennung begonnen.

Es gaben:

I. 0,250 Substanz 0,2350 Grm. Wasser und 0,713 Grm. Kohlensäure.

II. 0,217 Grm. Substanz 0,2015 Grm. Wasser und 0,6165 Grm. Kohlensäure.

III. 0,231 Grm. Substanz 0,2130 Grm. Wasser und $0,6510 \mathrm{Grm}$. Kohlensäure. 
Hieraus ergeben sich die Werthe:

$\begin{array}{ccc}\text { I. } & \text { II. } & \text { III. } \\ \text { p.C. } & \text { p.C. } & \text { p.C. } \\ \mathrm{C}=\mathbf{7 7 , 7 8} & \mathbf{7 7 , 4 8} & \mathbf{7 6 , 8 6} \\ \mathrm{H}=\mathbf{1 0 , 4 4} & \mathbf{1 0 , 3 1} & \mathbf{1 0 , 2 5}\end{array}$

Die Analysen sind in der Reihenfolge angegeben, in der sie ausgeführt sind; vielleicht ist die fortgehende Erniedrigung des $\mathrm{C}$ und $\mathrm{H}$ gehaltes durch die bei dem öfteren Oeffnen der Röhre unvermeidliche Einwirkung des Sauerstoffs bedingt.

Von den eigenthümlichen Säuren der trocknenden Oele sind meines Wissens nur die Leinölsäure und die isomere oder mit dieser identische Mohnölsäure gut untersucht. Die Zusammensetzung, welche Schüler und $\mathrm{Ou}$ demans für diese Säuren gefunden haben, weicht beträchtlich von der der obigen Säure, die ich Axinsäure nennen werde, $a b$; übereinstimmend sind sie nur in dem Punkte, dass die Atomzahl des Wasserstoffs niedriger ist, als die des Kohlenstoffs. Es ist mir bei der ausserordentlich schnell fortschreitenden Oxydation der Axinsäure durch Berührung mit atmosphürischer Luft nicht möglich gewesen, Salze dieser Säure zu gewinnen, die Aufstellung einer Formel für dieselbe ist somit nicht ausführbar, und zwar um so weniger als auch die Glycerinverbindung der Axinsäure, welche im Age enthalten ist, mit Laurostearin zu sehr verunreinigt ist, als dass die obigen $A$ nalysen einen hinlänglich genauen Ausweis über die Zusammensetzung des Axinsäureglycerins geben könnten. Nimmt man an, wie es auch Schüler im ähnlichen Falle gethan hat, dass die Atomzah! des Sauerstoffs 4 ist wie bei den nicht trocknenden Fettsäuren, so würde nach obigen Analysen die wahrscheinlichste Formel der Axinsäure sein $\mathrm{C}_{36} \mathrm{H}_{28} \mathrm{O}_{4}$, und die hieraus berechnete procentische Zusammensetzung: $\mathrm{C}=78,25, \mathrm{H}=\mathbf{1 0 , 1 4}$ p.C. Ob diese Formel die richtige ist, hoffe ich bestimmen zu können, wenn mir mehr Age zur Hand sein wird. Jedenfalls geht aus den obigen

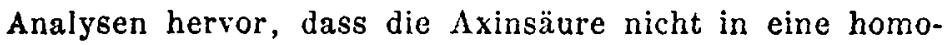
loge Reihe mit der Leinölsäure zu stellen ist. 
Bei der Verseifung des Age mit alkoholischer Kalilösung wurde nach Abscheidung der Säuren aus der wässrigen Flüssigkeit durch Neutralisiren, Abdampfen und Extrahiren mit Alkohol eine hinreichende Quantität Glycerin erhalten; dass aber nicht allein die Laurylsäure, sondern auch die Axinsäure in Verbindung mit Glycerin im Age enthalten ist, zeigt auch, abgesehen von den Verschiedenheiten in der Heftigkeit der Einwirkung des Sauerstoffs und der übrigen Eigenschaften beider Körper, noch der im Age gefundene höhere Kohlenstoffgehalt. Dieser letztere ist nur durch die Annahme erklärt, dass im Age die Verbindung von 3 Atomen Axinsäure mit 1 Atom Glycerin vorhanden ist. Nach der Berechnung würde diese verlangen $\left(3\left(\mathrm{C}_{36} \mathrm{H}_{27} \mathrm{O}_{2}\right), \mathrm{C}_{6} \mathrm{H}_{5} \mathrm{O}_{6}\right): \mathrm{C}=78,98$ p.C., $\mathrm{H}=9,93$ p.C., somit etwas höheren $\mathrm{C}$ gehalt und niedrigeren II gehalt, als die oben angeführten Analysen ergeben haben. Da jedoch das Age ausser diesem Triaxin auch Laurostearin $(\mathrm{C}=74,3, \mathrm{H}=11,2$ p.C. $)$ enthält, so kann es nicht wunderbar erscheinen, dass die angegebene Differenz $\mathrm{zwischen}$ den beobachteten und berechneten Werthen sich zeigt. Eine Trennung des Triaxin im Age vom Laurostearin zu bewerkstelligen erschien bei der geringen zu Gebote stehenden Quantität nicht wohl ausführbar.

Es ist bereits oben erwähnt, dass die Axinsäure durch den Sauerstoff sehr schnell verändert wird, schneller als man es selbst an dem Age sieht. Auch die Axinsäure zeigt bei dieser Oxydation bedeutende Aufnahme von Sauerstoff und dadurch bedingte Gewichtsvermehrung. Zur Untersuchung dieses Verhaltens wurden einige Tropfen Axinsäure über Quecksilber in trocknes Sauerstoffgas gebracht und 12 Tage lang damit in Berührung gelassen, die Säure schien dabei völlig erstarrt zu sein, und es liess sich dann das rückständige Gas leicht rein in ein anderes Absorptionsrohr überfüllen. Die Verhältnisse erschienen jedoch für eine quantitative Bestimmung der Sauerstoffaufnahme besonders deswegen sehr ungünstig, weil nicht allein die erstarrende Säure der Quecksilberoberfläche beim allmählichen Steigen des Niveaus in der Röhre eine unregelmässigre Gestalt gab, sondern auch die erhärtende 
oberflächliche Schicht der Säure das Innere vor der Einwirkung des Sauerstoffs schützte. Ungefïhr $0,3 \mathrm{Grm}$. Axinsäure (aus dem gehildeten Aginin herechnet, siehe unten) absorbirten 5,1 C.C. von $0^{\circ}$ und $1 \mathrm{Mm}$. Druck oder $0,0097 \mathrm{Grm}$. Sauerstoff; hiernach war also die Gewichtszunahme nur 3 p.C. unter der Annahme, dass nicht etwa Kohlensäure oder andere Gase noch bei dieser Oxydation ausgeschieden würden. Diess letztere \%eigte sich in der That bei der Prüfung des nach Beendigung der Einwirkung übergefüllten Gases, indem eine eingebrachte Kalikugel nur eine höchst kleine Verminderung des Volumen hervorrief, die 1 p.C. des Gasgemenges kaum überstieg und eben so wohl durch Anwesenheit von Wasserdampf als Kohlensäure bedingt sein honnte. Ich habe diess Verhalten jetzt nicht weiter untersucht, da zur Beurtheilung der Processe eine nähere Kenntniss derjenigen festen Stoffe nöthig ist, welche bei dem Erstarren der Axinsäure gebildet werden. Wic sehr aber bei diescm Versuche die erhärtende oherflächliche Schicht das Innere des öligen Körpers von der Einwirkung des Sauerstoffs geschützt hatte, ergiebt der folgende Versuch. $1,459 \mathrm{Grm}$. Axinsäure wurden einige Tage der Einwirkung der atmosphärischen Luft ausgesetzt, dabei aber öfter durch $\Lambda$ ufgiessen einiger Tropfen Aether die noch unzersetzte Säure der Einwirkung der Luft wieder zugänglich gemacht, dann bei $40^{\circ}$ die erstarrte Masse getrocknet. Das Gewicht der Substanz betrug jetz. $\mathbf{0 , \mathbf { j } 2 2} \mathrm{Grm}$., so dass also eine Gewichtszunahme von 13,7 p.C. erfolgt war.

Die Producte, welche die Oxydation der Axinsäure liefert, sind, wie es bereits rom $\Lambda$ ge angegeben ist, theils unlöslich in Wasser, Alkohol und Acther, theils löslich in den beiden letzteren. Die unlösliche Substanz scheint ein neuer Körper zu sein, für den ich den Namen Aginin vorschlage; der in Aether oder Altiohol lösliche Stoff ist wohl identisch mit der bereits bekannten Ilypoyaesaure, welche Gössmann*) im Erdnussöl entdeckt hat.

* Gössmann und Scheren, Anu. der Cheın. u. I'ham. $\mathrm{XCIV}, 230$. 
Das Aginin durch $\Lambda$ cther möglichst gereinigt, dann getrocknet bildet ziemlich spröde, leicht pulverisirbare Krusten, ist amorph, ohne Zersetzung nicht schmelzbar, von hellgelber Farbe, sehr hygroskopisch. Auf $\mathbf{8 0}^{\circ}$ erhitzt beginnt es sich sowohl in atmosphärischer Luft als auch in Wasserstoffgas unter Braunfärbung zu zersetzen, das Pulver klebt dabei etwas zusammen, es entwickeln sich Wasserdämpfe und farbiose Gase, welche von Kalilauge absorbirt werden. Da die Kalikugel hierbei gelb gefärbt wird und einen aromatischen Geruch annimmt, ohne dass das Quecksilber sich irgend wie verunreinigt zeigt, scheint zugleich eine vielleicht kleine Quantität einer gasförmigen organischen verbindung zu entstehen, welche durch Kali zerset\%t wird. Mit Wasser in ein Glasrohr geschmolzen und auf $110^{\circ}$ erhitzt bildete das Aginin eine dunkelbraune Lösung, in welcher einzelne braune Flocken schwammen. Durch Aetzalkalien wird es schon in der Kälte braun gefärbt und gelöst, durch Säuren wird in dieser Lösung ein flockiger Niederschlar gebildet. Durch Salpetersäure wird es unter Entwickelung salpetriger Säure beim Kochen gelöst, Wasser im Ueberschuss fällt aus dieser Lösung einen farblosen Körper in mikroskopischen Nadeln.

Die lebhafte Gasentwickelung, welche das Aginin schon unter $100^{\circ}$ beim Erhitzen zeigt, erschwerte die Analyse dieses Körpers sehr wesentlich. Die Verbrennungen gaben folgende Werthe:

I. 0,3515 Grm. Substanz gab 0,199 Grm. Wasser und $0,674 \mathrm{Grm}$. Kohlensäure.

II. 0,256ว Grm. Substanz gab 0,171ว̃ Grm. Wasser und $0,5672 \mathrm{Grm}$. Kohlensäurc.

III. 0,25000 Grm. Substanz gab 0,1690 Grm. Wasser und $0,5520 \mathrm{Grm}$. Kohlensäure.

IV. 0,3243 Grm. Substanz gab 0,2130 Grm. Wasser und 0,7022 Grm. Kohlensäure.

V. 0,2935 Grm. Substanz gab $0,1935 \mathrm{Grm}$. Wasser und $0,6340 \mathrm{Grm}$. Kohlensäure.

VI. 0,2470 Grm. Substanz gab 0,1640 Grm. Wasser und $0,5410 \mathrm{Grm}$. Kohlensäure.

Journ. f. prakt. Cbemie. IXXXX. 2. 
VII. 0,2005 Grm. Substanz gab 0,1310 Grm. Wasser und $0,4290 \mathrm{Grm}$. Kohlensäure.

VIII. 0,23/5 Grm. Substanz gab 0,1415 Grm. Wasser und $0,5110 \mathrm{Grm}$. Kohlensäure.

Diese Werthe ergeben:

\begin{tabular}{rrrrrrrr}
$\mathrm{I}$ & \multicolumn{1}{c}{$\mathrm{II}$} & \multicolumn{1}{c}{ III. } & \multicolumn{1}{c}{ IV. } & V. & VI. & VII. & VIII. \\
$\mathrm{H}=\mathbf{5 9 , 5 8}$ & $\mathbf{6 0 , 3 1}$ & $\mathbf{6 0 , 2 1}$ & $\mathbf{5 9 , 0 5}$ & $\mathbf{5 8 , 9 4}$ & $\mathbf{5 9 , 7 4}$ & $\mathbf{5 8 , 3 5}$ & $\mathbf{5 9 , 4 3}$ \\
$\mathbf{7 , 1 7}$ & $\mathbf{7 , 4 3}$ & $\mathbf{7 , 5 1}$ & $\mathbf{7 , 3 0}$ & $\mathbf{7 , 3 2}$ & $\mathbf{7 , 3 8}$ & $\mathbf{7 , 2 6}$ & $\mathbf{6 , 7 0}$
\end{tabular}

Es zeigen sich bei der Vergleichung dieser Zahlen sowohl hinsichtlich des Kohlenstoff- als auch hinsichtlich des Wasserstoffirehaltes nicht unerhebliche Differenzen, obwohl bei der Ausführung der Analysen keine Crsachen dieser Verschiedenheiten bemerkt wurden und gerade in einigen Analysen geringer $\mathrm{C}$ und II gehalt gefunden wurde, bei welchen die anfingliche Gasentwickelung aus der Substanz ziemlich ruhig vor sich gegangen war. Das $\Lambda$ ginin war vor der Analyse stets längere Zuit über Schwefelsäure im Vacuum bei gewöhnlicher Temperatur setrocknet. Die wahrscheinlichste Ursache der Verschiedenheit sind Verunreinigungen des Aginin durch fremde Stoffe, von denen man es so lange wohl nicht völlig wird befreien können, als man kein Mittel besitzt, das Aginin zu lösen oder init andcren Stoffen zu verbinden, ohne es zu zersetzen. Das zu den Analysen verwendete $\Lambda$ ginin war theils direct aus dem Age theils aus der $\Lambda$ xinsäure gewonnen; offenbar ist der aus beiden erhaltene Körper in Eigenschaften und Zusammensetzung derselbe. 0,45̃ Grm. Axinsäure lieferte bei mörrlichst weitgehender Zersetzung 0,270 Grm. Aginin oder $\mathbf{5 8 , 8}$ p.C. der zersetzten Säure.

Die hei der Finwirkung des Sauerstoffs auf die $\Lambda$ xinsïure neben dem $A$ ginin entstehende in Alkohol oder Aether lösliche Substan\% hatte eine hellgelbe Farbe, ist bei gewöhnlicher 'Temperatur fest, schmilzt und erstarrt schon bei $3 \tilde{5}^{\circ}$, so class die Erwïrmung mit der Hand sic vollkommen flüssig macht, zcrsetzt sich besonders beim öfteren Umschmelyen an der Luft unter $\Lambda$ bscheidung brauner Flocken ron harziger Beschaffenheit, und zeigt dabei cinen etwas ranzirren Geruch. Beim Erkalten heiss gesüttigter alkoholischer Lösung erhält man sic in sehr 
voluminösen Massen, welche aus Krystallblättchen bestehen, deren Faltungen unter dem Mikroskope das Bild von Nadeln simuliren, und beim Trocknen auf dem Filter erhält man einen seideartig glänzenden Ueberzug des Filters. In wässrigen Alkalilösungen ist die Fettsäure leicht löslich, und diese Lösung giebt mit alkalischen Erdsalzen Niederschläge, welche die Säure an die alkalische Erde gebunden enthalten. Durch salpetersaures Silberoxyd entsteht ein voluminöser Niederschlag, welcher das Silbersalz. der Säure enthält, der Niederschlag schwärzt sich aber allmählich schon bei gewöhnlicher Temperatur, noch schneller beim Erwärmen. 0,2625 Grm. des Silbersalzes über Schwefelsäure bei etwa $15^{0}$ getrocknet gab $\mathbf{0 , 0 8 2 5}$ Grm. Silber. Die alkoholische Lösung der Säure mit Aetzammoniak übersättigt, dann mit essigsaurem Baryt verset7.t, gab beim Erkalten über Aetzkalk einen voluminösen Niederschlag, welcher schnell abfiltrirt aus heissen Alkohol umkrystallisirt und dann noch mit Wasser gewaschen wurde. Das Wasser nahm hierbei wieder Baryt auf und die Analyse der von Wasser nicht gelösten Barytverbindung gab folgende Werthe:

$0,226 \mathrm{Grm}$. Substanz gab 0,191 Grm. Wasser, 0,4989 Grm. Kohlensäure und $0,069 \mathrm{Grm}$. schwefelsauren Baryt. Nach diesen Werthen würde die Zusammensetzung in 100 Gewichtstheilen sein:

$$
\begin{array}{lc}
\text { Beobachtet. } & \begin{array}{c}
\text { Berechnct } \\
\left(\mathrm{C}_{32} \mathrm{H}_{22} \mathrm{BaO}_{4}\right) .
\end{array} \\
\mathrm{C}=60,21 & \mathbf{5 9 , 7 3} \\
\mathrm{H}=\mathbf{9 , 3 9} & \mathbf{9 , 0 2} \\
\mathrm{Ba}=\mathbf{1 8 , 4 7} & \mathbf{2 1 , 3 3}
\end{array}
$$

Die Analyse der Säure selbst ergab:

I. 0,281 Grm. Substanz gab 0,3035 Grm. Wasser und 0,777 Grm. Kohlensäure.

II. 0,287 Grm. Substanz gab $0,3005 \mathrm{Grm}$. Wasser und 0,795 Grm. Kohlensäure.

Dic Säure zeigtc sonach die Zusammensetzung:

I.

C 75,41

$\mathrm{H} \quad 12,00$

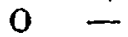

11.

75,55

11,62
Berechnet

$\left(\mathrm{C}_{32} \mathrm{H}_{30} \mathrm{O}_{4}\right)$.

7丂.วิ9

11.81

12,60 
Wenn nach diesen Analysen nun auch die Silberverbindung mehr, die Barytverbindung weniger Basis ergeben hat, als die Rechuung nach der Formel der Salze der Hypogiasüure verlangt, so stimmt doch einerseits der Schmelıpunkt und die übrigen Eigenschaften sehr genau mit den Angahen Gössmann's über die llypogäsäure üherein, und auch die Analysen der Siure selbst gaben hierınit ziemlich gut übereinstimmende liesultate. Die Zcrsetzlichkeit der Hypogäsäure, welche Gössmann bereits geschildert hat, und welche den Gliedern der Angelicasiuregruppe im Allgemeinen cigen ist, bewirkt sicherlich, dass bei der Bildung der Hypogäsäure aus der $\Lambda x i n-$ sïure auch ein Theil derselben sich bereits weiter zersetzt, und hierin mag die Ursache des zu niedrig gefundenen Baryumgehaltes in obiger Analyse des Barytsal\%es zu suchen sein. Viclleicht wird dic Glycerinverbindung der Hypogäsäure, die sich hei der Zersetzung des Age neben dem Aginin bildet, der Einwirkung des Sauerstoffs besser widerstehen als die freic Säure; die zu Gebote stehende Quantitä derselben war jedoch für diege Untersuchung zu gering. Die Analyse des neben Aginin aus dem Age gebildeten Fettes ergab folgendes:

I. 0,257 Grm. Substanz gab 0,252 Grm. Wasser und 0,689 Grm. Kohlensäure.

II. 0,230 Grm. Substanz gab 0,225 Grm. Wasser und 0,614 Grm. Kohlensäure.

Hiernach ergieht sich die Zusammensetzung:

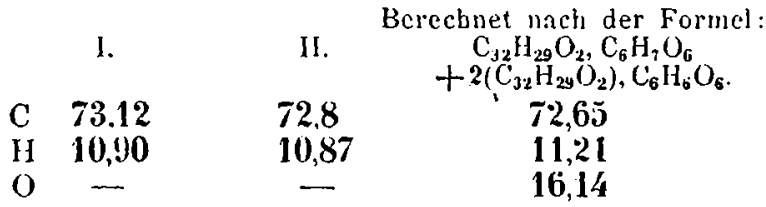

Nach diesen analytischen Ergebnissen kann das entstehende Glycerid (und ein solches ist es nach allen seinen Eigenschaften) nicht die Verbindung von 3 Atomen Säure mit 1 Atom Glycerin sein, denn eire solche Verhindung würde $\mathbf{C} \mathbf{7 6 , 5}$ und $\mathbf{H} \mathbf{1 1 , \tilde { 3 }}$ der Rechnung nach verlangen. auch das Monohypogaeïn und Dihypogaeïn liegen ein jedes 
in seinem $\mathrm{Cgehalte} u m$ Procente von den gefundenen Werthen entfernt, die Beimengung von Laurostearin aber, welche aus dem Ige in dieses fragliche Fett übergeht, könnte den C'gehalt nur etwas erhöhen, wenn das Fett Monohypogäin wäre, da es mit dem Dihypogäin nahezu gleichen Cgehalt besitzt; es bleibt demnach kein anderer Ausweg übrig, als anzunehmen, dass das bei der Oxydation des Triaxin gebildetc Fett ein Gemenge gleicher Atome Mono- und Di-Hypogiiin sei, und hierfür ergicbt sich auch noch eine weitere Bestätigung, Aus den obcn erwähnten Versuchen ging hervor, dass aus $100 \mathrm{Th}$. Axinsäure 58,8 Th. Aginin und 54,9 Th. Hypogäsäure entstanden. Wollte man nun annehmen, dass aus jedem Atome Axinsäure ein Atom Hypogäsäure entstünde, so würde man die Formel der $\Lambda$ xinsäure auf $\mathrm{C}_{54} \mathrm{H}_{42} \mathrm{O}_{6}$ oder gar $\mathrm{C}_{72} \mathrm{H}_{56} \mathrm{O}_{8}$ erhöhen müssen, um ein Atom Hypogäsäure daraus entstehen $\mathrm{zu}$ lassen. Schon die Formeln würden diese Erhöhung verlangen. Es ist daher das Wahrscheinlichste, dass bei der Oxydation der Substanz aus je 2 Atomen Axinsäure 1 Atom Hypogäsäure entsteht, und ist diess der Fall, so wird aus 2 Atomen Triaxin 1 Atom Monohypogäin und 1 Atom Dihypogäin gebildet werden, wie es auch die Analysen oben zeigten.

Die Processe des Trocknens von Leinöl und anderer trocknender Oele scheinen ganz analoge Verhältnisse zu bieten; bei einigen vorläufigen Proben mit getrocknetem Leinöl ergab sich, dass auch hier ein Fett neben einem bröcklichen in Aether unlöslichen Körper entstebt.

Weitere Mittheilungen über diese Zersetzungen so wie über die Oxydationsproducte des Age behalte ich mir noch vor. 